\title{
REFORMA SANITARIA, SALUD PÚBLICA Y BIENESTAR BIOLÓGICO DURANTE LA INDUSTRIALIZACIÓN ESPAÑOLA: EL CASO DE ALCOY, 1840-1915¹
}

\author{
José Joaquín García-Gómez \\ Departamento de Economía y Empresa - Facultad de Ciencias Económicas y Empresariales. Universidad de Almería \\ josejgg@ual.es \\ Javier Puche \\ Departamento de Estructura e Historia Económica y Economía Pública - Facultad de Economía y Empresa. Universidad de Zaragoza. \\ jpuche@unizar.es
}

Recibido: 15 noviembre 2014; Aceptado: 5 junio 2015.

Cómo citar este artículo/Citation: García-Gómez, José Joaquín y Javier Puche (2016), “Reforma sanitaria, salud pública y bienestar biológico durante la industrialización española: el caso de Alcoy, 1840-1915”, Asclepio 68 (1): p128. doi: http://dx.doi.org/10.3989/ asclepio.2016.07

RESUMEN: En este artículo analizamos los efectos que la reforma sanitaria tuvo sobre el bienestar biológico y la salud de las poblaciones españolas durante el proceso de industrialización. Examinamos el caso de Alcoy, una de las ciudades pioneras de la industrialización española. Los presupuestos municipales en salud pública y los datos de estatura de los reemplazos militares constituyen las principales fuentes para su estudio. Los resultados muestran que los comienzos de la reforma sanitaria, a finales del siglo XIX, supusieron una mejora no sólo de las condiciones ambientales y de salubridad de la ciudad, sino también del estado de salud de su población residente. Hasta que los políticos liberales no incrementaron las dotaciones presupuestarias en el campo de la salud pública y las infraestructuras urbanas, Alcoy sufrió los lastres iniciales del urban penalty con secuelas negativas que se manifestaron directamente en el aumento de la morbi-mortalidad y la caída de la estatura. Los datos sugieren una relación positiva entre políticas activas de salud pública y bienestar biológico.

PALABRAS CLAVE: Reforma Sanitaria; Salud Pública; Bienestar Biológico; Industrialización Española; Alcoy.

\section{SANITARY REFORM, PUBLIC HEALTH AND BIOLOGICAL WELFARE DURING SPANISH INDUSTRIALIZATION: THE CASE OF ALCOY, 1840-1915}

ABSTRACT: This article analyses the effects of the sanitary reform on the biological welfare and the Health of the Spanish population during the industrialization process. Examines the case of Alcoy, one of the pioneer hubs of the Spanish industrialization. The main sources of the study are the municipality budgets of Public Health and the stature of the conscripts. Results show that the starting of the sanitary reform, in the late 19th, meant not only an improvement of the conditions and the salubrity of the city, but of the resident's state of health. Until the liberal politicians did not increase the public health and the urban infrastructures assignments on the Budgets, Alcoy suffered the initial problems of the urban penalty, with negative effects that were revealed directly in the increasing morbi-mortality and the falling stature. Data suggest a positive correlation between active public health politics and biological welfare.

KEY WORDS: Sanitary Reform; Public Health; Biological Welfare; Spanish Industrialization; Alcoy.

Copyright: (C) 2016 CSIC. Este es un artículo de acceso abierto distribuido bajo los términos de la licencia Creative Commons Attribution (CC BY) España 3.0. 


\section{INTRODUCCIÓN}

Los estudios sobre los efectos causados por la primera revolución industrial en el bienestar biológico y la salud de las poblaciones son ya numerosos (Steckel y Floud, 1997; Komlos, 1998; Haines, 2004; CinnireIla, 2008; Komlos y Küchenhoff, 2012; Baten y Blum, 2014). Con excepciones puntuales, la historia antropométrica ha mostrado que durante los primeros estadios de la industrialización la talla de las poblaciones urbanas fueron más cortas que la de las poblaciones rurales hasta comienzos del siglo XX (Komlos, 1998). Las ciudades eran, por tanto, lugares menos saludables para vivir. La denominada urban penalty fue sustancial durante los comienzos de la industrialización (Huck, 1995; Haines, 2004; Voth, 2004). No obstante, existieron algunas excepciones a esta penalización, destacando los casos de las ciudades de Munich (Baten, 2001), de la provincia de Lieja en Bélgica (Alter, Neven y Oris, 2004) y Lisboa (Reis, 2009).

Desde finales del siglo XIX, y a lo largo del siglo XX, la situación se revirtió. Como resultado de la puesta en marcha de las reformas sanitarias, traducido en un aumento del gasto en políticas de salud pública desde finales del ochocientos, las poblaciones urbanas comenzaron a disfrutar de mejores servicios médicosasistenciales e infraestructuras urbanas, condiciones que contribuyeron a que los habitantes de las ciudades comenzaran a tener unas tallas medias más elevadas que las de las poblaciones rurales. En la primera mitad del siglo XX los avances de la salud pública en las ciudades y zonas urbanas se reflejaron en la dinámica de una acelerada reducción de la morbi-mortalidad, fundamentalmente infantil. Esto último discurrió paralelo al avance de los procesos de transición epidemiológica (caída de la importancia de las enfermedades ambientales e infecciosas transmitidas por el agua y los alimentos), sanitaria y nutricional (Szreter, 1988; Bernabeu y Barona, 2011; Martínez-Carrión, 2012; Hatton, 2013; Barona, 2014).

Este artículo analiza el impacto que la reforma sanitaria tuvo sobre el bienestar biológico y la salud de las poblaciones españolas durante la primera industrialización. Examina para ello el caso de Alcoy, una de las ciudades pioneras de la industrialización española en el siglo XIX, y cuyo crecimiento industrial se fundamentó en la doble especialidad en la producción de tejidos de lana y la manufactura papelera (Aracil y García-Bonafé, 1974; Calatayud, 2001; Martínez Galarraga, 2009). Aunque en los últimos años el caso de Alcoy ha sido objeto de varios estudios para analizar los efectos que su proceso de industrialización tuvo sobre las condiciones de vida y salud de su población (Beneito, 2003; Puche, 2009; García-Gómez, 2013, 2015), este artículo supone un salto cualitativo que cubre un vacío historiográfico ante la escasez de trabajos nacionales sobre las consecuencias que las reformas sanitarias tuvieron sobre el bienestar biológico de las poblaciones urbanas. Por ello, y en consonancia con lo observado en la literatura especializada (Szreter, 1988, 1997), planteamos la hipótesis que los comienzos de la reforma sanitaria en Alcoy, a finales del siglo XIX, supusieron una mejora no sólo de las condiciones ambientales y de salubridad de la ciudad, sino también del estado de salud de su población residente. Hasta que los políticos liberales no incrementaron las dotaciones presupuestarias en el campo de la salud pública y las infraestructuras urbanas, Alcoy sufrió los lastres iniciales del urban penalty con secuelas negativas que se manifestaron directamente en el aumento de la morbi-mortalidad y la caída de la estatura.

El artículo se estructura en cinco apartados. Además de esta introducción, el apartado dos describe las fuentes de estudio, los datos y la metodología utilizada. En el siguiente, en el apartado tres, se sitúa y analiza la reforma sanitaria de Alcoy en el contexto internacional de la primera industrialización. Esta sección servirá de introducción a aquellos que por primera vez se acercan al tema. Posteriormente, en el apartado cuatro, se analiza el impacto que la reforma sanitaria tuvo en los niveles de vida biológicos de la población de Alcoy a partir de la evolución de la estatura media de los mozos nacidos en la ciudad entre 1856 y 1915 y la morbi-mortalidad. El estudio finaliza con las conclusiones, sintetizadas en el apartado cinco.

\section{FUENTES, DATOS Y METODOLOGÍA}

Los gastos municipales realizados por el Ayuntamiento de Alcoy en materia de salud pública, los datos de estatura y de alegaciones físicas formulados por los mozos de los reemplazos militares y las tasas de morbimortalidad infantil constituyen las fuentes principales de este estudio. La evolución de las haciendas locales durante la etapa liberal apenas ha llamado la atención hasta la fecha de historiadores y economistas, a pesar de que el análisis desagregado de los presupuestos municipales y de otros instrumentos fiscales de las políticas públicas son esenciales para desvelar los objetivos y, sobre todo, las realizaciones prácticas que llevaron a cabo los políticos liberales. Con datos de los presupuestos elaborados y aprobados por el Ayuntamiento de Alcoy y las liquidaciones finalmente 
ejecutadas en materia de salud pública, hemos construido la evolución que siguió el gasto público en reforma sanitaria durante gran parte de la etapa liberal, entre $1836-1840$ y $1911-1914^{2}$. Sobre este punto, no obstante, varias observaciones deben ser realizadas: primera, hasta el ejercicio presupuestario de 1855 la fuente no distinguía entre presupuestos y liquidaciones, por lo que entre 1836 y 1854 sólo se puede hablar de contabilidad, pudiéndose únicamente hacer una estimación aproximada sobre la asignación de recursos en materia de salud pública; segunda, a partir de 1855 y hasta 1903 encontramos ya diferenciados presupuestos y liquidaciones, con las únicas excepciones de los ejercicios de 1858 y 1873; tercera, entre la década de 1880 y 1903, además del presupuesto ordinario, fue habitual e inevitable elaborar uno adicional y, en muchos ejercicios, otro extraordinario. La razón principal estriba en que con el ordinario no se cubrían los gastos de cada ejercicio. A partir de 1904 desaparecieron los presupuestos adicionales y los extraordinarios; y cuarta, desde 1878 el Ayuntamiento de Alcoy presentó los presupuestos y las liquidaciones de los ensanches de la ciudad. Debido a la importancia que tuvo esta partida en la reforma sanitaria, hemos sumado los presupuestos y las liquidaciones de los gastos de los ensanches a los correspondientes de cada ejercicio desde ese año (García-Gómez, 2013).

Para analizar el impacto que, en el contexto de la primera industrialización, tuvo la reforma sanitaria sobre el bienestar biológico de la población en Alcoy, utilizamos dos tipos de datos: los registros de estatura de los mozos alcoyanos que fueron Ilamados a filas provenientes de los reemplazos militares (Puche, 2009); y los datos de las alegaciones físicas que fueron presentadas por algunos quintos para eludir legalmente, de acuerdo con el cuadro de exenciones físicas establecido por el Ejército español, el servicio militar, y que fueron excluidos finalmente por dicha alegación. La estatura, junto a otros indicadores ya conocidos, como la mortalidad infantil y la esperanza de vida, es un excelente indicador del nivel de salud de las poblaciones. Aunque la genética condiciona en un $80 \%$ la estatura de una persona, la talla es muy sensible al consumo de necesidades básicas (alimentación y asistencia sanitaria) y al impacto de la morbilidad (Silventoinen, 2003; Steckel, 2008; Martínez-Carrión, 2012; Hatton, 2013). La serie de estatura media de Alcoy arranca con el reemplazo de 1876 y finaliza con el de 1936, correspondiente a las generaciones nacidas entre 1856 y 1915 . Entre ambos periodos, aunque más concretamente desde finales del siglo XIX, se sitúa la reforma sanitaria de Alcoy que nos permite examinar las relaciones entre el gasto municipal en salud pública, como indicador de la reforma sanitaria, y durante el proceso de industrialización.

La muestra antropométrica está compuesta por 16.102 tallas de los 18.632 mozos que fueron alistados en Alcoy entre 19 y 21 años desde 1876 hasta 1936. Representa el $86,4 \%$ del total de mozos alistados. El 13,6\% restante no se presentó al proceso de reclutamiento por diferentes causas (fallecimiento, enfermedad, emigración, prófugo...). Como la ciudad de Alcoy experimentó un intenso proceso industrializador desde la década de 1830 , ello provocó a su vez un gran aumento de su población gracias a la inmigración, que procedió sobre todo de las poblaciones rurales de su entorno. La fuente de quintas nos informa sobre las migraciones. Se detalla el lugar de nacimiento del mozo inmigrante pero no la edad de llegada al municipio de destino. En consecuencia, no podemos conocer en qué entornos (mundo rural o mundo urbano) crecieron y cómo pudieron influir las distintas condiciones ambientales en las principales etapas del crecimiento (infancia y estirón adolescente). Para eludir este obstáculo, y probado que la talla de los mozos inmigrantes tuvo una influencia sobre la tendencia secular de la estatura en Alcoy en algunos periodos (Puche, 2009), en este artículo vamos utilizar únicamente los promedios de talla de los mozos nacidos en Alcoy. De esta manera, podremos analizar con mayor exactitud qué consecuencias tuvo la puesta en marcha de la reforma sanitaria en las condiciones de salud y nutrición de las sucesivas cohortes de reclutas nacidos en la ciudad. Por este motivo y no otro, la serie de estatura de Alcoy comienza con el reemplazo de 1876, ya que es el primer año en que la fuente de quintas comenzó a informar del origen de procedencia del mozo. Los datos de lugar de nacimiento que acompañan a los de la talla son regulares entre los reemplazos de 1876-1899 y 1912-1936, con un corte de información entre las quintas de 1901-1911.

Además de la estatura, este artículo analiza también la distribución y la evolución porcentual de las alegaciones físicas que fueron presentadas por algunos mozos para eludir legalmente el servicio militar. Se trata de una variable muy interesante pero apenas utilizada en los estudios españoles de historia antropométrica, ya que refleja las condiciones sanitarias y el estado de salud durante los años del crecimiento de las sucesivas cohortes. Para evitar las situaciones de fraude, en este trabajo solo hemos considerado a aquellos mozos que fueron declarados excluidos del 
servicio militar por impedimento físico y que presentaron alegaciones físicas. De este modo, se ha llevado a cabo un análisis de las enfermedades alegadas en función de su etiología, utilizando los estudios realizados por Thomas Mckeown a través de su propuesta de clasificación de las enfermedades, que distingue, básicamente, entre enfermedades infecciosas y no infecciosas (Mckeown, 1978). Así, se ha elaborado una clasificación de enfermedades, basada en su propuesta, que nos permitirá analizar no solo los procesos morbosos, sino también el cambio en el patrón epidemiológico y la influencia que tuvo la reforma sanitaria durante los procesos de industrialización y transición demográfica.

Para finalizar, debemos señalar que los datos de estatura media no han sido estandarizados a la edad de 21 años. Como ha sido ya apuntado en varios estudios, desde la década de 1850 los reemplazos militares en España fueron sorteados a edades homogéneas que cambiaron según las necesidades del ejército ${ }^{3}$. Al ser este trabajo un estudio de caso, con menores observaciones de talla que otros basados en macro-muestras regionales (Martínez-Carrión y Moreno-Lázaro, 2007; Puche, 2011; Ramón-Muñoz, 2011; Ayuda y Puche, 2014), presentamos los datos sin estandarizar considerando que los cambios producidos en la edad de reclutamiento influyeron por igual en todos los mozos alistados. Por ello, las estaturas se presentan respetando los periodos en los que el reclutamiento se produjo a distintas edades: 19, 20 y 21 años.

\section{LA REFORMA SANITARIA DE ALCOY EN EL CONTEXTO DE LA PRIMERA INDUSTRIALIZACIÓN}

Los estudios históricos-sociales y de la medicina, así como los de antropometría y demografía histórica, han mostrado que a mediados del siglo XIX, en plena era industrial, las zonas urbanas eran lugares más insalubres para vivir, especialmente las ciudades industriales (Harris, 1994; Bourdelais, 1997; Floud, Fogel, Harris y Hong, 2011, 2014). La presión demográfica, el hacinamiento y las pésimas condiciones de las viviendas, los efectos contaminantes de las fábricas, las duras condiciones de trabajo infantil, la existencia de talleres y minas mal protegidas, junto con las desventajas del abastecimiento precario de proteínas y nutrientes básicos, como carne, huevos y leche, provocaron efectos devastadores sobre la salud infantil y de los trabajadores adolescentes en el medio urbano (William y Mooney, 1994; Voth, 2004; Martínez-Carrión, Puche y Cañabate, 2013). En países industrializados, como Estados Unidos, Gran Bretaña
- Alemania, los datos de altura y mortalidad revelan el fuerte deterioro que experimentó el nivel de vida biológico en las zonas urbanas frente a un medio rural más saludable en términos relativos en las décadas centrales del ochocientos (Huck, 1995; Komlos, 1998; Haines, 2004; Voth, 2004; Cinirella, 2008). La existencia de una posible penalización urbana, sin embargo, variaba según el tamaño y las condiciones ambientales de las ciudades y la extensión de las actividades industriales y manufactureras (Foster, Chinn y Rona, 1983; Humphries y Leuning, 2009; Martínez-Carrión, Pérez-Castroviejo, Puche y Ramon-Muñoz, 2014).

Desde finales del siglo XIX, y una vez superadas las secuelas más negativas del industrial-urban penalty, las poblaciones de las zonas urbanas comenzaron a disfrutar de mayor bienestar biológico que las poblaciones rurales. La disminución de la contaminación industrial, la presencia de mejores servicios médicosasistenciales, la mejora de las infraestructuras urbanas y del suministro de alimentos frescos (particularmente leche), así como la reducción de la adulteración de los alimentos procesados, fueron factores que ayudaron a reducir la elevada morbi-mortalidad y que posibilitaron que la desventaja urbana desapareciera sobre la rural en los albores del siglo XX. El descubrimiento de la refrigeración mecánica y la reducción de los costes de transportes a finales del siglo XIX, por ejemplo, facilitaron la manipulación higiénica, el almacenamiento y el consumo de productos perecederos de alto valor nutritivo, como la leche. La oferta de leche digestible fue muy importante en los mercados urbanos, especialmente en las ciudades más populosas ${ }^{4}$. Con todo, las mejoras más importantes procedieron de las condiciones sanitarias y de vivienda (Millward y Baten, 2010). Diversos estudios han evidenciado que las reformas sanitarias tuvieron efectos muy positivos, contribuyendo a reducir las elevadas tasas de mortalidad, particularmente de mortalidad infantil a finales del siglo XIX y comienzos del XX, momento en que la mejora de las infraestructuras urbanas y los servicios médicos favorecieron los progresos de las poblaciones del mundo urbano (Floud, Fogel, Harris y Hong, 2011). Así se ha observado para los casos de Estados Unidos, Gran Bretaña y Gales (Bell y Millward, 1998; Cage y Foster, 2002; Cutler y Miller, 2005; Costa, 2013).

En el contexto europeo, el caso de Alcoy proporciona otro ejemplo de cómo las incipientes políticas de salud pública puestas en marcha ayudaron a mejorar las condiciones de vida y salud de sus habitantes desde comienzos del siglo XX. Alcoy, ciudad 
grande e industrializada, sufrió a mediados del siglo XIX las consecuencias de la industrial-urban penalty por los efectos de la transición de las actividades protoindustriales al sistema de fábrica y el impacto medioambiental provocado por el rápido proceso de urbanización. Ambos procesos tuvieron un impacto muy negativo sobre el bienestar biológico y la salud de la población alcoyana (Puche, 2009). En el tránsito del siglo XIX al XX, la situación comenzó a mejorar paulatinamente como resultado de la reducción de la mortalidad por la menor incidencia de las enfermedades infecciosas y las epidemias, la disminución del trabajo infantil $y$, en general, por los progresos alcanzados en las condiciones de salubridad junto al desarrollo de las infraestructuras urbanas higiénicosanitarias. Este avance de las condiciones de vida y salud discurrió paralelo al aumento del gasto municipal en políticas de salud pública.
El Estado puede contribuir al crecimiento económico y a la salud mediante el empleo de recursos para la provisión de bienes públicos, como educación, sanidad e infraestructuras. Si éstos contribuyen a esos objetivos y los agentes privados no están en condiciones de proveerlos, será necesario que el Estado gaste más. Las investigaciones de García-Gómez $(2013,2015)$ y García-Gómez y Salort (2014, pp. 103-108) nos permite disponer, a nivel local, de información relevante sobre la evolución del gasto público llevado a cabo por el Ayuntamiento de Alcoy en materia de reforma sanitaria entre mediados del siglo XIX y el primer cuarto del siglo XX (Tabla 1). Para evitar el efecto distorsionador de la inflación, los datos han sido deflactados utilizando el índice de precios al consumo estimado por Maluquer (2006, pp. 333-382), obteniendo seis series. En la segunda columna se observa la evolución que tuvo el presupuesto total del municipio durante el periodo de

Tabla 1. Gasto público del Ayuntamiento de Alcoy en la reforma sanitaria, 1836-1840 / 1911-1914

\begin{tabular}{|c|c|c|c|c|c|c|c|}
\hline & $\begin{array}{l}\text { Presupuesto } \\
\text { total } \\
\text { (1) }\end{array}$ & $\begin{array}{l}\text { Presupuesto } \\
\text { liquidado } \\
\text { (2) }\end{array}$ & $\begin{array}{l}\text { Presupuesto } \\
\text { total en } \\
\text { reforma } \\
\text { sanitaria } \\
\text { (3) }\end{array}$ & $\begin{array}{c}\% \\
(3 / 1)\end{array}$ & $\begin{array}{l}\text { Presupuesto } \\
\text { liquidado } \\
\text { en reforma } \\
\text { sanitaria } \\
\text { (4) }\end{array}$ & $\begin{array}{c}\% \\
(4 / 2)\end{array}$ & $\begin{array}{l}\text { Reforma } \\
\text { sanitaria } \\
\text { liquidada } \\
\text { per cápita }\end{array}$ \\
\hline $1836-1840$ & $57.541,98$ & $57.541,98$ & $7.357,45$ & 12,79 & $7.357,45$ & 12,79 & 0,39 \\
\hline $1841-1845$ & $135.109,06$ & $135.109,06$ & $49.989,56$ & 37,00 & $49.989,56$ & 37,00 & 2,50 \\
\hline $1846-1850$ & $182.356,34$ & $183.712,00$ & $42.879,28$ & 23,51 & $45.029,22$ & 24,51 & 2,08 \\
\hline $1851-1855$ & $98.659,68$ & $90.065,17$ & $39.380,74$ & 39,92 & $40.019,42$ & 44,43 & 1,70 \\
\hline $1856-1860$ & $185.313,82$ & $221.015,41$ & $58.321,01$ & 31,47 & $58.684,66$ & 26,55 & 2,33 \\
\hline $1861-1865$ & $211.312,25$ & $195.073,16$ & $50.115,43$ & 23,72 & $43.891,39$ & 22,50 & 1,66 \\
\hline $1866-1870$ & $177.688,55$ & $128.674,29$ & $41.812,25$ & 23,53 & $33.540,06$ & 26,07 & 1,20 \\
\hline $1871-1875$ & $492.055,17$ & $346.340,79$ & $48.501,36$ & 9,86 & $37.425,32$ & 10,81 & 1,20 \\
\hline $1876-1880$ & $592.651,24$ & $461.061,51$ & $61.911,56$ & 10,45 & $57.254,43$ & 12,42 & 1,78 \\
\hline $1881-1885$ & $806.051,19$ & $602.448,97$ & $55.307,20$ & 6,86 & $43.865,97$ & 7,28 & 1,41 \\
\hline $1886-1890$ & $942.683,45$ & $655.702,24$ & $100.528,39$ & 10,66 & $41.952,68$ & 6,40 & 1,39 \\
\hline 1891-1895 & $848.181,87$ & $549.468,24$ & $130.291,92$ & 15,36 & $84.420,33$ & 15,36 & 2,75 \\
\hline $1896-1900$ & $737.484,61$ & $401.410,90$ & $126.010,94$ & 17,09 & $99.270,09$ & 24,73 & 3,18 \\
\hline 1901-1905 & $1.159 .002,38$ & $815.849,90$ & $129.335,53$ & 11,16 & $110.925,34$ & 13,60 & 3,40 \\
\hline 1906-1910 & $893.406,00$ & $907.003,45$ & $220.582,47$ & 24,69 & $233.768,63$ & 25,77 & 6,97 \\
\hline 1911-1914 & $1.032 .662,01$ & $1.093 .269,98$ & $374.627,95$ & 36,28 & $390.918,44$ & 35,76 & 11,33 \\
\hline
\end{tabular}

Fuente: García Gómez (2013) y García Gómez y Salort (2014, p. 105). 
estudio, mientras que en la tercera tenemos el presupuesto finalmente liquidado en cada uno de los quinquenios considerados. En la cuarta columna tenemos las cifras del gasto presupuestado para la reforma sanitaria. El dato es relevante porque aporta información relativa sobre la predisposición que los políticos liberales tuvieron en avanzar en dicha reforma. La quinta columna, por su parte, muestra el porcentaje que representaba el presupuesto total en reforma sanitaria respecto al presupuesto total. En la sexta y séptima columna, respectivamente, se ofrecen las cantidades y los porcentajes que finalmente fueron liquidadas en esta materia y, por tanto, el gasto público realmente realizado por la Administración liberal. Finalmente, la octava columna muestra el porcentaje de gasto per cápita en la reforma sanitaria. Para su cálculo hemos estimado la población de hecho de Alcoy de cada año basándonos en los datos de los censos de población del Instituto Nacional de Estadística (INE) y, a su vez, en la tasa media anual de crecimiento acumulativo entre los años para los que disponíamos de cifras de población. Analizaremos en primer lugar el gasto municipal liquidado en reforma sanitaria (columna 6).

Observamos que durante las décadas de 1840 y principios de la de 1850, el gasto público realizado en materia de reforma sanitaria es significativo, en torno al 35 por ciento del presupuesto total liquidado, si bien los niveles de gasto público eran bajos. Hay excepciones, como la del quinquenio de 1836-40, pero está vinculada al control presupuestario que hizo el Ayuntamiento liberal. En el periodo de $1856-60$ a $1876-80$ se aprecia una tendencia clara al descenso (19 por ciento de media) que se sostendrá hasta finales de la década de 1880, cuando alcanza su mínimo histórico (6,4 por ciento). Habrá que esperar al decenio de 1890 y primer quinquenio del siglo $\mathrm{XX}$, aproximadamente, para detectar un cambio de tendencia, ahora alcista (18 por ciento), y que se intensifica fuertemente desde 1906 hasta 1914 (30 por ciento de media). Este cambio de tendencia está relacionado con las primeras etapas del reformismo político del régimen de la Restauración, bajo los gobiernos regeneracionistas de Maura y Canalejas (diputado nacional por la circunscripción de Alcoy), y con la presión del movimiento obrero. Prueba de este incremento del gasto público es que desde la década de 1890 se produjo un acercamiento progresivo de los gastos liquidados en reforma sanitaria a los gastos inicialmente presupuestados, de forma que incluso en el periodo de los quinquenios 1906-10 y 1911-14 los primeros excedieron a los segundos, generando un déficit presupuestario que no se había producido en toda la segunda mitad del siglo XIX.
Si consideramos ahora la evolución del gasto per cápita en la reforma sanitaria, podemos detectar la casi absoluta falta de implicación de los políticos liberales alcoyanos en asuntos de salud pública hasta entrada la década de 1890 (columna 8). Es cierto que hay unos primeros esfuerzos en los albores del Estado liberal, pero muy tímidos. El gasto per cápita en reforma sanitaria aumentará a partir de 1891 y explotará, esencialmente, desde 1906. Como en el caso del gasto liquidado, con el cambio de siglo, el regeneracionismo y el despliegue del movimiento obrero forzaron a la Administración local a aumentar el gasto en salud pública e infraestructuras higiénicos-sanitarias. La tendencia inaugurada con el siglo se mantendrá, al menos, durante la década de 1910, que recibirá un buen espaldarazo.

En suma, la provisión pública del Ayuntamiento de Alcoy en el ámbito de la reforma sanitaria no siguió una tendencia clara entre mediados del siglo XIX y el primer cuarto del siglo XX. Fuera ya por motivos económicos o ideológicos, como la insuficiencia crónica de recursos de la Hacienda local liberal o los cánones liberales de la época, se ha detectado que el desinterés de los políticos liberales en Alcoy por la sanidad se prolongó hasta finales del siglo XIX, teniendo efectos muy negativos sobre las condiciones de vida y salud de la mayoría de la población. Sería con la llegada del nuevo siglo, y con él el reformismo de la segunda etapa de la Restauración y las reivindicaciones de la clase trabajadora, cuando el gasto público en salud pública e infraestructuras urbanas comenzó a incrementarse. Proseguirá durante los años de la I Guerra Mundial.

\section{RESULTADOS Y ANÁLISIS}

\subsection{Reforma sanitaria y estatura}

En este apartado se relaciona el bienestar biológico con la reforma sanitaria en Alcoy durante el proceso de industrialización. Con ello, se desea analizar las relaciones que se establecen entre el gasto público en sanidad e infraestructuras higiénicos-sanitarias y uno de los principales indicadores del nivel de vida biológico, la estatura.

Entre las cohortes de $1836-40$ y 1911-15 la estatura media de los mozos alistados en Alcoy creció unos 3 centímetros. Los mayores logros de la estatura, sin embargo, se alcanzaron durante las generaciones nacidas en el primer cuarto del siglo XX. La tendencia secular del crecimiento de la talla no fue lineal, ya que se vio interrumpida por un ciclo de deterioro que afectó fundamentalmente a las cohortes nacidas en- 
tre $1866-70$ y $1876-80$ según los resultados de la serie analizada. Durante este periodo la altura promedio de los quintos experimentó una caída de un centímetro, coincidiendo con la industrialización del textil alcoyano, la intensificación de la urbanización y el adelanto de la edad legal de reclutamiento de 20 a 19 años, factor este último que pudo influir también en el descenso de la estatura media (Puche, 2009).

Desde la década de 1820 en Alcoy se fue consolidando el sistema fabril y la mecanización en las primeras fases del proceso productivo, cardado e hilado. El proceso de mecanización, aunque incompleto, supuso una notable disminución de los costes y de los precios de venta. Los precios más bajos estimularon una mayor demanda en el mercado agrario interior, por definición pobre e inestable, aunque también se vio beneficiada por la protección arancelaria. Así, el aumento de la demanda favoreció el de la producción lanera. En este contexto de efervescencia industrial, y en contra de lo que cabría esperar, la estatura disminuyó, señal inequívoca de un empeoramiento de las condiciones de vida y salud de la población. Así se desprende del contraste realizado entre el número de husos mecánicos inscritos en la matrícula industrial y la evolución de la talla en Alcoy (Gráfico 1). Aunque este ejercicio es arriesgado por el carácter restringido de la muestra de estaturas utilizado (cohortes masculinas), el análisis presenta resultados interesantes. De la figura se deduce que mientras el número de husos mecánicos creció desde las décadas de 1850-1860, reflejo del avance de la mecanización en la hilatura y la industrialización, la estatura bajó, síntoma de la caída del bienestar biológico. Para los que vivieron la coyuntura 1865-80, se puede decir que experimentaron mejoras económicas asociadas a la expansión industrial, pero percibieron en sus cuerpos las secuelas de la malnutrición y la infección como consecuencia de la intensidad del trabajo manufacturero a edades tempranas y las pésimas condiciones de trabajo.

Gráfico 1. Estatura media anual y husos mecánicos en Alcoy, cohortes de nacimiento de 1860-1915

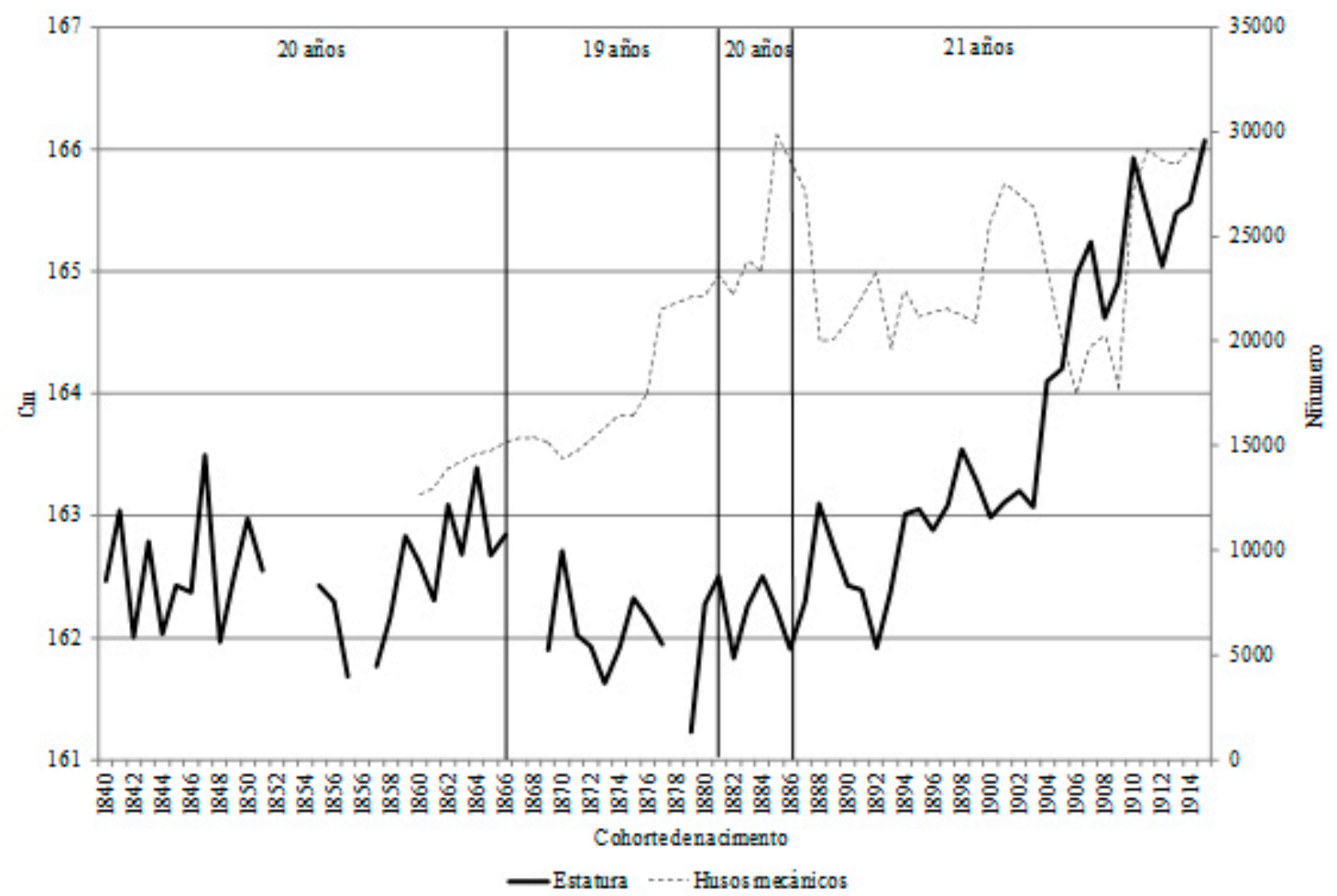

Fuente: los datos de estatura media anual en Puche (2009). Los datos de husos mecánicos en la matrícula industrial de Alcoy en Martínez Galarraga (2009, pp. 108-110). 
Aparte de los efectos negativos iniciales causados por la industrialización, otros factores que explicarían la caída de la estatura alcoyana en la etapa 186680 apuntarían al impacto medioambiental provocado por el fuerte crecimiento demográfico y la rápida urbanización. En las décadas centrales del siglo XIX, y como resultado de la elevada inmigración rural que llegó de la comarca, la población de Alcoy pasó de 20.924 habitantes en 1835 a 32.497 en 1877, cifras que suponen un aumento superior al $50 \%$ y un incremento anual del $1,31 \%$, que podemos calificar de elevado ${ }^{5}$. Estos movimientos migratorios comportaron la intensificación de la urbanización y revelaron los graves problemas urbanísticos que presentaba la ciudad $^{6}$. La urbe creció al borde de la saturación y recibió a miles de emigrantes sin las infraestructuras necesarias para acogerlos. El hacinamiento y la proliferación de barrios mal equipados y con viviendas precarias caracterizaron el urbanismo de aquellos años. Como analizaremos en el próximo apartado, esta conjunción de factores contribuyó a aumentar la morbi-mortalidad, la cual, a su vez, afectó a la estatura de las sucesivas cohortes. La congruencia con los datos de gasto público y gasto per cápita liquidado en reforma sanitaria es muy elevada. Los Gráficos 2 y 3 lo reproducen respectivamente para el período de las cohortes de $1856-60$ y 1911-15.

La comparación realizada entre ambas series sugiere que la tendencia de la estatura en Alcoy fue muy sensible a la evolución del gasto municipal en políticas de salud pública durante el proceso de industrialización. El Gráfico 2 muestra que hubo una alta correlación entre la inversión pública en reforma sanitaria y las condiciones de salud y nutrición de los mozos alcoyanos. Observamos que cuando la primera disminuyó entre 1856-60 y 1886-90, los promedios de talla cayeron y viceversa. Esto último ocurrió a partir de las

Gráfico 2. Estatura y gasto liquidado en reforma sanitaria en Alcoy, cohortes de nacimiento de 1856-60 / $1911-15$. Medias quinquenales

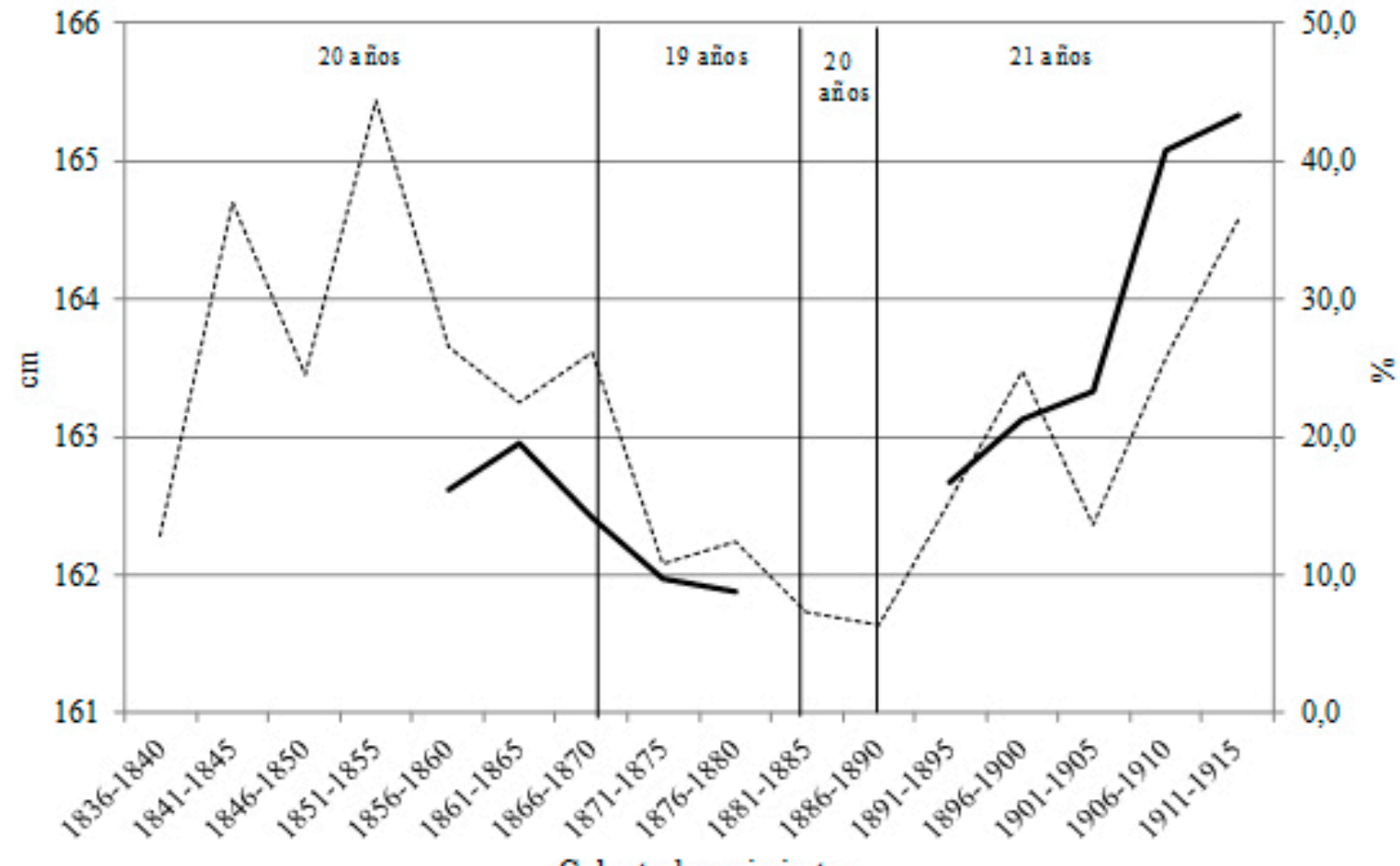

Cohorte de nacimiento

\section{— Estatura (nacidos en Alcoy) …... Gasto liquidado en reforma sanitana}

Fuente: los datos de estatura en Puche y Cañabate (2015). Los datos de gasto liquidado en reforma sanitaria en Alcoy en García Gómez (2013) y García Gómez y Salort (2014, p. 105). 
Gráfico 3. Estatura y gasto per cápita en reforma sanitaria en Alcoy, cohortes de nacimiento de 1856-60 / 1911-15. Medias quinquenales

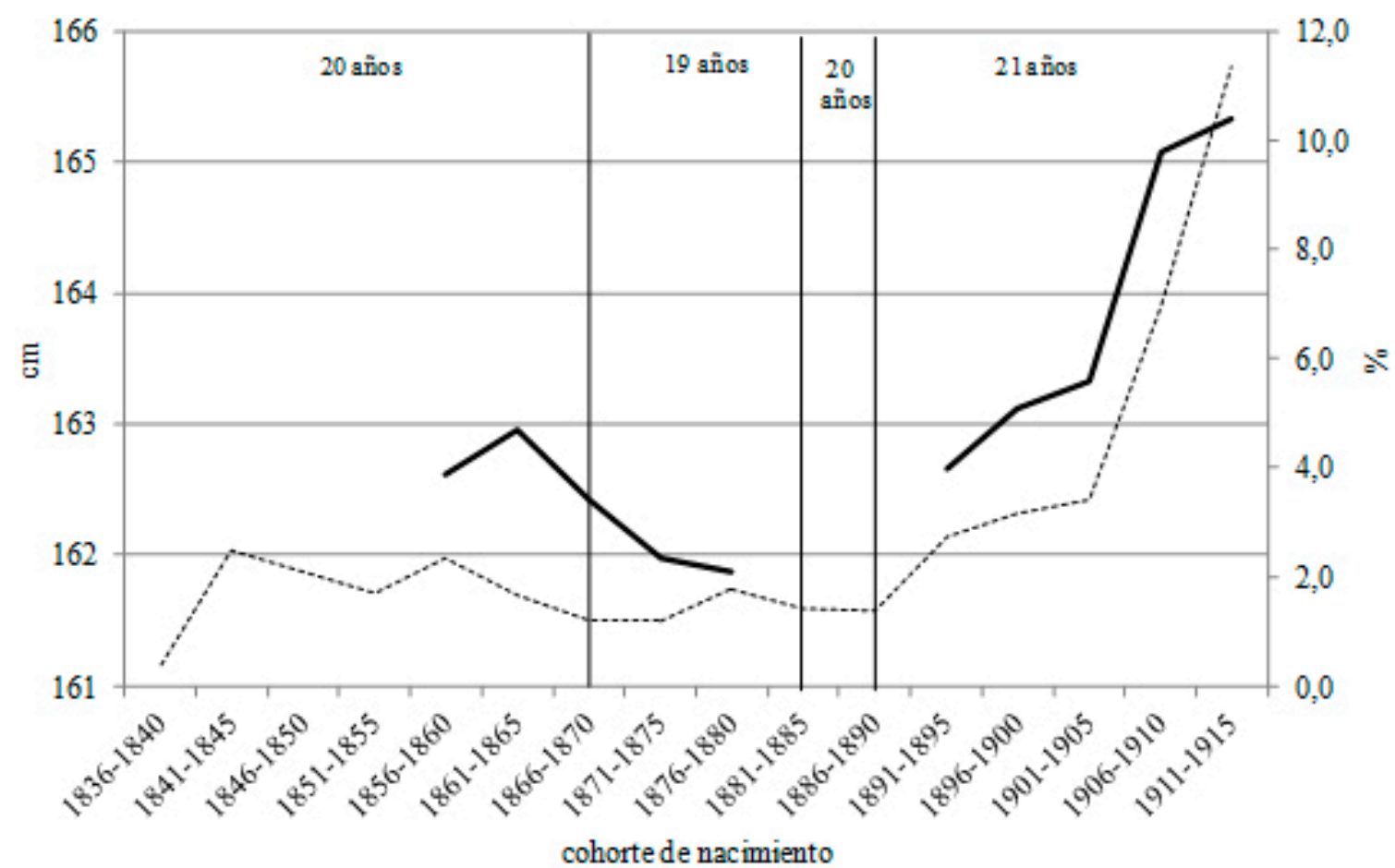

\section{—Estatura (nacidos en Alcoy) $\quad$-....... Gasto per cápita en reforma sanitaria}

Fuente: los datos de estatura en Puche y Cañabate (2015). Los datos de gasto per cápita en reforma sanitaria en Alcoy en García Gómez (2013) y García Gómez y Salort (2014, p. 105).

cohortes de la década de 1890 , donde se observa que el aumento de la estatura corrió pareja al aumento del gasto público en sanidad e infraestructuras urbanas. Esta asociación entre lo que se invirtió y los resultados en estatura es también evidente en el caso del gasto per cápita en reforma sanitaria, según se desprende del Gráfico 3. A más gasto por habitante, la estatura media fue mayor.

Según el dictamen que elaboró la Junta Local de Sanidad en 1868 para mejorar las condiciones higiénicas de la ciudad, los mayores inconvenientes que presentaba Alcoy eran los problemas urbanísticos, el hacinamiento y el abocado de las aguas inmundas en pozos ciegos. Como consecuencia del intenso proceso industrializador, a mediados del siglo XIX la construcción de edificios fue por detrás de la creciente demanda de viviendas. Esta situación provocó que los barrios más populosos soportaran una alta densidad de población, llegando a ser en algunos casos superior a los 2.500 habitantes per ha. Esta cifra, escandalosa, revelaba que el hacinamiento era la norma y que el peligro de enfermar estaba casi asegurado. La carencia de infraestructuras higiénicos-sanitarias agravaba aún más las condiciones de insalubridad en que vivía la mayoría de la población, especialmente las clases trabajadoras. El caso del servicio de alcantarillado lo ejemplifica.

Hacia 1820 la red de alcantarillado era prácticamente inexistente en Alcoy. En esa década el Ayuntamiento propuso un proyecto para su construcción que, sin embargo, años después no había sido ejecutado por la falta de financiación. A finales de los años cuarenta, la Alcaldía sacaba a subasta su construcción, cuya red inicial partiría del Ayuntamiento y recorrería las calles del Mercado Vell y de la Barbacana, cobrando un canon a todos aquellos vecinos que quisieran conectar sus aguas residuales y pozos ciegos a la red general. Esta medida, no obstante, no resolvió el problema 
sanitario: por un lado, porque la mayoría de la población, residente en los barrios obreros, quedaba desatendida; y por otro, porque muchos habitantes, al ver el precio elevado que había que pagar por el servicio, no se acogieron a la mejora. De esto modo, hacia 1850 solamente había 64 solicitudes de conexión a la red; de ellas 22 correspondían a la zona donde vivía la población más acomodada de la ciudad. Dado que el objetivo principal era luchar contra la costumbre vecinal de tirar los residuos fecales, así como los excrementos de las caballerías en las calles en cualquier hora del día, en 1850 el Ayuntamiento publicó un bando que restringía la extracción de los primeros desde la madrugada hasta el mediodía. Pero esta medida fue criticada por el gremio de labradores que manifestaba su total oposición:

Sin abono no hay cosechas y sin cosechas no pueden pagarse los arriendos de las tierras (...). Desde inmemorial es costumbre sacar las materias fecales en todo tiempo del año hasta el mediodía y a todas horas el estiércol de las cuadras (Beneito, 2003, p. 43).

El edicto municipal subrayaba las pésimas condiciones de vida y de salubridad que existían en la ciudad. Consciente de esta situación y de que los presupuestos municipales eran insuficientes para cubrir los gastos de este servicio público, en 1860, el Ayuntamiento de Alcoy decidió que fuera la iniciativa privada la que emprendiera las obras. Poco a poco, a lo largo de las décadas finales del siglo XIX y comienzos del siglo XX, la ciudad fue dotándose de un servicio de alcantarillado ${ }^{7}$. Este caso demuestra que en Alcoy la provisión pública de infraestructuras higiénicas-sanitarias fue bastante deficiente al comienzo del proceso de industrialización y de la rápida urbanización. Eso no significa, sin embargo, que hubiera desinterés por parte de las autoridades locales. En 1883, la Junta Local de Sanidad mostró una especial preocupación por mejorar la higiene pública, denunciando repetidamente la existencia de estercoleros en las casas. Las medidas de salubridad adoptadas, no obstante, chocaban con una resistencia generalizada: en primer lugar, con el propio Ayuntamiento, que no disponía de los recursos económicos suficientes para emprender mejoras urbanísticas; en segundo lugar, con el gremio de labradores, que veía en peligro el estiércol que había de servir como abono natural para mejorar la productividad de sus tierras en cultivo; en tercer lugar, con la mayoría de los habitantes que no quería o no podía pagar el canon del servicio de alcantarillado; y en cuarto y último lugar, con un número significativo de pequeños industriales y artesanos que capturaban las aguas residuales y los líquidos sobrantes de las fuentes para mover las ruedas de los molinos o para enjuagar las pieles enderezadas, actividades que provocaban no pocas filtraciones, humedades y malos olores, además de un número elevado de enfermedades infecciosas, principalmente respiratorias.

A partir de la segunda mitad del siglo XIX, el Ayuntamiento de Alcoy, al igual que aconteciera con otros municipios de su entorno geográfico más próximo (Salort, 2008; Pascual Artiaga y Trescastro, 2013), comenzó a promulgar una serie de reglamentos y ordenanzas municipales dirigidos a mejorar las condiciones higiénicas y sanitarias de la ciudad. Unos iban orientados a controlar el comercio de la carne y la calidad de los alimentos que se vendía en los mercados, otros a regular los servicios sanitarios y asistenciales, otros a mejorar la edificación de las viviendas, otros a reglamentar el suministro y la red de aguas públicas, etc. Pero la aprobación de esas normativas no fue suficiente para mejorar las condiciones ambientales y de salubridad de la urbe. Faltaba lo más importante, que se cumplieran, y en la mayoría de los casos no fue así. $A$ veces porque no había suficiente personal para hacer cumplir las disposiciones aprobadas; en otras porque la aplicación de la normativa no interesaba a ciertos colectivos por motivos particulares en cada caso, algunos apuntados ya, - arrendatarios y arrendadores, labradores, vaqueros, vendedores, etc. - ; y finalmente, y quizás sobre todo, porque el Ayuntamiento carecía del presupuesto para poder aplicar las mejoras proyectadas. Este factor, hasta los años finales del siglo XIX y comienzos del siglo $X X$, impidió que la alcaldía se implicase más en el fomento de las infraestructuras urbanas y la higiene pública para afrontar el drama de la elevada morbi-mortalidad. Pero no sólo eso. Este apartado ha mostrado que la caída progresiva del gasto municipal en salud pública entre las décadas de 1860 y 1880 tuvo también su réplica en el descenso de la estatura de los mozos alcoyanos, reflejo del empeoramiento que experimentaron las condiciones de vida y salud de la población en ese periodo. La situación comenzó a cambiar a partir de la década de 1890. Las causas son multifactoriales: disminución del trabajo infantil, mejoras en las condiciones de trabajo y vivienda, mejor dieta alimentaria y reducción de la morbi-mortalidad. Esto último, sin duda, fue debido al aumento del gasto público destinado a mejorar los servicios de limpieza y alcantarillado, el control de la potabilidad del agua, la higiene de los alimentos, etc. 


\subsection{Reforma sanitaria y morbi-mortalidad}

La literatura especializada ha revelado que el aumento del gasto en políticas de salud pública estuvo directamente relacionado con la caída de la mortalidad y el aumento de la esperanza de vida durante los siglos XIX y XX, entre otros factores (Szreter, 1988; Bell y Millward, 1998; Cutler y Miller, 2005) ${ }^{8}$. El objetivo de esta sección es explorar las relaciones entre la reforma sanitaria y las condiciones de vida y salud de la población de Alcoy, a través de la morbi-mortalidad. Aunque no disponemos de series anuales de morbilidad y mortalidad infantil en Alcoy, los datos disponibles muestran una relación estrecha con la evolución del gasto público en reforma sanitaria y la estatura durante el proceso de industrialización (García-Gómez, 2013, 2015; García-Gómez y Salort, 2014).

Para suplir la falta de información sobre morbilidad, hemos utilizado los datos de las alegaciones físicas que fueron presentadas por algunos mozos para in- tentar eludir legalmente el servicio militar de acuerdo con el cuadro de exenciones físicas establecido. Es una variable valiosa por cuanto nos informa del estado de salud de los jóvenes alistados y de las condiciones medioambientales. Considerando que muchos mozos intentaron evitar con engaño el servicio militar manifestando problemas físicos simulados, hemos computado únicamente a aquellos quintos que fueron declarados excluidos totales por las alegaciones físicas presentadas ${ }^{9}$, considerando dos grandes periodos: los reemplazos militares de 1860-99 y 1901-36. La Tabla 2 muestra el número de casos analizados y el porcentaje que representaba cada grupo de enfermedad, así como su evolución.

¿Qué grupos de enfermedades sobresalieron? ¿Estuvieron influidas por los efectos de la industrialización y la rápida urbanización? ¿Qué papel jugó la reforma sanitaria? Consideraremos en primer lugar el periodo 1860-99.

Tabla 2. Número y porcentaje de alegaciones físicas presentadas por los mozos excluidos en Alcoy, reemplazos de $1860-1899$ y $1901-1936$

\begin{tabular}{|c|c|c|c|c|}
\hline \multirow{2}{*}{ Grupo de enfermedades } & \multicolumn{2}{|c|}{$1860-1899$} & \multicolumn{2}{|c|}{$1901-1936$} \\
\hline & Casos & $\%$ & Casos & $\%$ \\
\hline Cardíaca & 8 & 2,5 & 1 & 0,1 \\
\hline Carencial & 27 & 8,4 & 29 & 1,9 \\
\hline Cerebral & 1 & 0,3 & & \\
\hline Cutánea & 20 & 6,3 & 7 & 0,5 \\
\hline Digestivo & 4 & 1,3 & 1 & 0,1 \\
\hline Fonador-auditivo & 25 & 7,8 & 12 & 0,8 \\
\hline Genital & 7 & 2,2 & 2 & 0,1 \\
\hline Genitourinario & 3 & 0,9 & 2 & 0,1 \\
\hline Malformación & 17 & 5,3 & & \\
\hline Mental & 2 & 0,6 & 12 & 0,8 \\
\hline Muscular-óseo & 85 & 26,6 & 744 & 48,4 \\
\hline Nervioso & 8 & 2,5 & 3 & 0,2 \\
\hline Ocular & 57 & 17,8 & 27 & 1,8 \\
\hline Respiratorio & 18 & 5,6 & 16 & 1,0 \\
\hline Sin especificar* & 38 & 11,9 & 682 & 44,3 \\
\hline Total & 320 & 100,0 & 1.538 & 100,0 \\
\hline
\end{tabular}

* En este grupo hemos agrupado las alegaciones genéricas de "defecto e inutilidad física".

Fuente: Expedientes de reemplazos de Alcoy. Elaboración propia. 
Tabla 3. Tasa de mortalidad infantil en Alcoy, 1875-1879 / 1928-1932 (\%о)

\begin{tabular}{ccc}
\hline & $\begin{array}{c}\text { Mortalidad infantil } \\
\text { (por 1.000 nacidos vivos) }\end{array}$ & $\begin{array}{c}\text { Mortalidad postneonatal } \\
\text { (11 meses siguientes) }\end{array}$ \\
\hline $1875-1879$ & 169 & 125 \\
$1898-1902$ & 131 & 97 \\
$1928-1932$ & 79 & 57 \\
\hline
\end{tabular}

Fuente: Beneito (2003, p. 36).

En general, los datos de la Tabla 2 conducirían a una visión pesimista, ya que los porcentajes de todos los grupos de enfermedades fueron más elevados en la segunda mitad del siglo XIX que en el primer tercio del siglo XX. Y lo mismo se observa con la tasa de mortalidad infantil (Tabla 3). Ambas evidencias sugerirían que los comienzos de la industrialización y la intensificación de la urbanización, a mediados del siglo XIX, tuvieron efectos negativos iniciales sobre la salud de los niños y jóvenes adolescentes alcoyanos. Durante ese periodo y hasta finales de la centuria, las dolencias musculares-óseas y oculares concentraban casi el $45 \%$ de las alegaciones físicas presentadas por los mozos excluidos. Le seguían, por este orden, las enfermedades carenciales, las relacionadas con el sistema fonador-auditivo, las enfermedades de la piel y las que afectaban al sistema respiratorio, representando un $28 \%$. La mayoría de ellas, como veremos a continuación, estuvieron muy influidas por las deterioradas condiciones laborales que los trabajadores, muchos de ellos niños, soportaron en la industria textil, papelera y metalúrgica.

Las condiciones ambientales y de trabajo que soportaban los obreros alcoyanos a finales del siglo XIX eran muy agresivas (Egea, 1984, pp. 133-139; Beneito, 2003, pp. 56-62). Así se desprende de la información oral que Miguel Gisbert trasladó al Ayuntamiento de la ciudad en virtud de la Real Orden de 5 diciembre de 1883, al lamentarse de: la falta de higiene que en general tienen nuestras fábricas, entre las que, a su parecer, se debe poner de relieve el hacinamiento en que se tiene al obrero por la estrechez de espacio (Beneito, 2003, p. 56).

Años después, en 1894, las actas de la Junta Local de Sanidad se expresaban en los mismos términos denunciando:

las condiciones especiales que reúnen los edificios industriales, como son, calor, humedad, hacinamiento en los obreros, escasa condición atmosférica, ventilación defectuosa... (Beneito, 2003, pp. 56-57).

La falta de espacio hacía que muchas máquinas no guardarán la distancia prudencial aconsejada, circunstancia ésta que provocaba que muchos operarios sufrieran cantidad de luxaciones y hernias ${ }^{10}$. En las fábricas de papel, por ejemplo, los niños solían ocuparse del martinete, mazo de gran peso destinado para picar y satinar las hojas de papel. La actividad era monótona pero muy peligrosa, ya que la mínima distracción pasaba a ser fatal para las manos de los niños y adolescentes que manipulaban esos martillos grandes. Muchos de ellos quedaban lisiados para toda la vida, situación que les impedía regresar al trabajo para ganarse el sustento y que les condenaba a ocupar los lugares peor pagados o, peor aún, a engrosar la larga lista de los mendicantes (Beneito, 2003, pp. 59-60).

Tabla 4. Evolución de las enfermedades infecciosas en Alcoy, 1875-1879 / 1928-1932 (\%)

\begin{tabular}{lccc}
\hline Causa & $\mathbf{1 8 7 5 - 7 9}$ & $\mathbf{1 8 9 8 - 1 9 0 2}$ & $\mathbf{1 9 2 8 - 3 2}$ \\
\hline Por transmisión aérea & 35,6 & 39,1 & 27,0 \\
\hline Por agua y alimentos & 14,9 & 12,6 & 9,1 \\
\hline Por microorganismos & 3,6 & 8,3 & 4,5 \\
\hline Total & 54,1 & 60,0 & 40,6 \\
\hline
\end{tabular}

Fuente: Beneito (2003, p. 266). 
Pero las dolencias musculares-óseas no fueron las únicas enfermedades profesionales. Destacaron también las alegaciones oculares, respiratorias y cutáneas. Como el núcleo de la actividad industrial de la ciudad eran las empresas textiles y papeleras, en ambas el agua, tratada en calderas, tenía un claro protagonismo en las fases de lavado y tintado de la lana y el papel. Sin embargo, muchas de estas instalaciones no fueron capaces de vaporizar el agua adecuadamente, lo que debió favorecer la proliferación de patologías de carácter bronquial, respiratorio y ocular (Tabla 4$)^{11}$. Las calderas expulsaban cantidad de vapor y los obreros trabajaban en talleres donde el calor y la concentración de polvos de lana y gases tóxicos eran muy elevados, afectando a los bronquios y a la vista de los operarios. Asimismo, el uso de productos químicos que se empleaba para la fabricación de papel y cerillas producían a menudo afecciones cutáneas, como eccemas, que daban lugar a costras y escamas, y tumores de piel (Beneito, 2003, pp. 57-58).

Además de las dolencias ya apuntadas, la Tabla 2 revela también que durante el periodo 1860-1899 algunos mozos alegaron problemas de desarrollo físico y que hemos agrupado en el grupo de enfermedades carenciales. Representa el $8,4 \%$ del total de las alegaciones físicas presentadas. Este porcentaje habría sido mayor si hubiéramos computado a los mozos excluidos directamente por su cortedad de talla. El dato, con todo, es significativo, ya que pone de manifiesto que la alimentación de una parte de los mozos alcoyanos era pobre en las primeras fases de la industrialización. La sobreexplotación de la mano de obra - cristalizado en largas jornadas de trabajo y bajos jornales-, el gravamen de los impuestos de consumos y los elevados precios de los artículos de primera necesidad, lo explicarían (Egea, 1984, pp. 139-140).

Como consecuencia del fuerte crecimiento demográfico que experimentó la ciudad, la demanda de alimentos básicos aumentó exponencialmente, desencadenándose un incremento de los precios relativos a los mismos ${ }^{12}$. Si a esta situación añadimos que los impuestos de consumo gravaban y encarecían el precio final de un buen número de artículos de primera necesidad, es fácil pensar que muchos habitantes de Alcoy experimentaran una disminución del consumo alimenticio, o al menos una disminución de la calidad de los nutrientes ingeridos (Beneito, 2003, p. 63). Este empeoramiento del estado nutricional tuvo su correlato en la caída de la estatura de los mozos llamados a filas (Puche, 2009).
Habrá que esperar al comienzo de siglo y al aumento del poder adquisitivo de los obreros para detectar cambios relativos en la dieta de los alcoyanos. Entre 1905 y 1915 el nivel de vida aumentó y algunos productos como la leche, los huevos, el azúcar o los embutidos comenzaron a hacerse más asequibles en la cesta de la compra de las familias trabajadoras. También se incrementó el uso del carbón para calentar las viviendas, circunstancia que a buen seguro aminoró el impacto del frio en las casas y las humedades, y se generalizó el consumo de jabón, avances que permitieron reducir la incidencia de la morbilidad (Beneito, 2003, p. 67). Los datos de la Tabla 2 lo constatan. Entre 1901 y 1936 el número y porcentaje de alegaciones físicas presentadas por los mozos alcoyanos excluidos por esta casuística cayeron en picado - a excepción de las dolencias muscularesóseas que se incrementaron ${ }^{13}-$. La correlación con el aumento del gasto público en reforma sanitaria, el incremento de la estatura y la caída de la mortalidad infantil y las enfermedades infecciosas es muy elevada (Gráficos 2 y 3 y Tablas 3 y 4).

\section{CONCLUSIONES}

A partir del ejemplo de la ciudad industrial de Alcoy en la segunda mitad del siglo XIX y comienzos del siglo XX, este artículo ha analizado la relación entre las inversiones en salud pública y el bienestar biológico durante el proceso de industrialización. Para ello el estudio ha tenido en cuenta tres indicadores: los presupuestos y las inversiones realizadas por el Ayuntamiento de Alcoy en la salud pública, los datos de estatura de los mozos alistados y, finalmente, las enfermedades y problemas físicos expuestas por los quintos excluidos del servicio militar y las tasas de mortalidad infantil. Los resultados alcanzados son relevantes, mostrando que, al menos, desde mediados del siglo XIX el gasto público en reforma sanitaria tuvo un peso determinante en el nivel de vida biológico de los habitantes de la ciudad, junto a las condiciones de alimentación y morbilidad.

Se ha observado que entre las décadas de 1860 y 1880 la caída del gasto municipal en salud pública tuvo su réplica en un aumento de la morbi-mortalidad y un deterioro de la estatura media de los mozos nacidos en Alcoy. El proceso de mecanización y modernización de la industria textil dejó sin trabajo a muchos campesinos de las poblaciones agrarias de la comarca, que vieron en la emigración a la ciudad la posibilidad de obtener unos ingresos que no podían conseguir en sus lugares de origen. Ello dio lugar, durante las déca- 
das centrales del siglo XIX, a un intenso movimiento migratorio, provocando a su vez un gran aumento de la población residente. Aunque los políticos locales eran sabedores de los problemas higiénico-sanitarios de los que adolecía la ciudad, el Ayuntamiento no disponía de los medios económicos para afrontarlos, por lo cual la provisión pública de infraestructuras necesarias para acogerlos fue deficiente. La existencia de viviendas pequeñas e insalubres, barrios masificados y los efectos de la industrialización trajo consigo un empeoramiento general de las condiciones de vida para la población alcoyana, especialmente la de menores ingresos. Estas duras condiciones, además, contribuyeron a que Alcoy sufriese numerosas epidemias y una alta incidencia de enfermedades infecciosas, destacando los altos porcentajes de fallecimiento por bronquitis y neumonías. Esta prevalencia está asociada no solo al hacinamiento y las pobres condiciones sanitarias que soportaba la ciudad, sino también a las pésimas condiciones de trabajo y nutrición que sufría la clase trabajadora. La incidencia del trabajo infantil, la intensidad del trabajo manufacturero, los bajos salarios y una alimentación pobre e insuficiente hicieron que muchos niños y adolescentes, operarios en los malolientes talleres textiles y papeleros, fueran presa fácil de las epidemias e infecciones, dejando secuelas en el tamaño de sus cuerpos. El resultado final fue la disminución de la altura media durante las décadas de 1860, 1870 y 1880.

A partir de la década de 1890 la situación comenzó a mejorar paulatinamente. Se constata que el incremento de las inversiones y la normativa higiénicasanitaria efectuadas por el Ayuntamiento de Alcoy tuvieron una influencia importante en las condiciones de vida de la población. Ambas acciones contribuyeron tanto al descenso de la morbi-mortalidad infantil como al aumento de la estatura media de los mozos en el corto y medio plazo. Así ocurrió desde 1895 hasta 1915 , cuando las tasas de morbilidad y mortalidad infantil y las alegaciones físicas presentadas por los quintos empezaron a descender mientras los desembolsos en materia de salud pública e infraestructuras urbanas eran cada vez más cuantiosas. Esto último, sin duda, tuvo también un efecto positivo en las mejoras que experimentaron las condiciones de vivienda y de saneamiento, junto a la disminución del trabajo infantil y las mejoras relativas en la dieta alimentaria y los niveles de vida en general.

En suma, con este estudio se ha demostrado no solo la relación positiva entre gasto sanitario y bienestar biológico, sino también la importancia que tienen las políticas de salud pública para procurar unas mejores condiciones de vida y salud a las poblaciones.

\section{NOTAS}

1. Este artículo se ha realizado en el marco de los proyectos de investigación ECO2012-33286, HAR2013-47182-C2-2-Py ECO2015-65582, financiados por el MEC-MICINN (Gobierno de España) y del proyecto de investigación 19512/PI/14 financiado por la Fundación Séneca (Agencia de Ciencia y Tecnología de la Región de Murcia). Una versión preliminar del mismo fue presentado en la I Conference European Society of Historical Demography (ESHD), celebrado en Alghero (Italia), 25-27 de septiembre de 2014. Por último, agradecemos a los evaluadores anónimos los comentarios recibidos.

2. Las partidas presupuestarias que afectaban a la salud pública y, por tanto, a la reforma sanitaria en Alcoy eran las siguientes: gastos en médicos, comadronas, beneficencia y hospital, los de aguas, limpieza, arbolado y parques, mercados, cementerios, infraestructuras urbanas básicas (alcantarillado, calles, cañerías, fuentes y matadero), estudios urbanísticos (esta partida incluía gastos relativos a la planificación, desarrollo, reforma y ampliación de los edificios y espacios de la ciudad), caminos, Junta de Reformas Sociales y personal que trabaja en este organismo, principalmente (García-Gómez, 2013; García-Gómez y Salort, 2014, p. 103-104).

3. Los reclutamientos se hicieron con quintos de 20 años entre los reemplazos de 1858 a 1885 (primer reemplazo); de 19 años desde el segundo reemplazo extraordinario de 1885 a 1899; de 20 años, de nuevo, entre 1901 y 1905; y, finalmente, de 21 años a partir de 1907. No hubo reemplazos en 1900 ni en 1906, para ajustarse a la legislación que incrementó las edades de reclutamiento y sorteo a 20 y 21 años, respectivamente (Ayuda y Puche, 2014, pp. 103-104).

4. Según el estudio de Craig, Goodwin y Grennes (2004, p. 325), la adopción de la refrigeración mecánica a finales del siglo XIX en Estados Unidos incrementó el consumo de lácteos un $1,7 \%$ y la ingesta proteínica un 1,25\% anual después de la década de 1890. Como resultado, y de acuerdo con sus estimaciones, la refrigeración contribuyó directamente al menos a un $5,1 \%$ del incremento de la estatura media que experimentaron las cohortes post-refrigeración. Si a ello le añadimos los efectos indirectos asociados con las mejoras en la calidad de los nutrientes y la reducción de las enfermedades, el impacto global fue considerablemente mayor.

5. La tasa de crecimiento anual de la población española durante la primera mitad del siglo XIX fue de $0,63 \%$ y se redujo en la segunda a 0,43\% (Carreras y Tafunell, 2010, p. 31). 
6. Por estos años, Alcoy se asentaba entre barrancos y ríos, por lo que la existencia de terreno edificable era reducido.

7. En 1894, una memoria sobre las condiciones higiénicas de Alcoy denunciaba que algunos tramos de la red de alcantarillado estaba en pésimas condiciones y dado que no tenía suficiente pendiente, en algunas zonas había casi medio metro de defecaciones en descomposición que solamente se limpiaba cuando llovía (Beneito, 2003, p. 45).

8. Para el caso español ver Salort (2008).

9. Al acto de clasificación y declaración de soldados asistían por ley, además de los talladores, miembros de ayuntamiento y representantes militares, un médico titular encargado de prácticas los reconocimientos médicos a todos los mozos alistados. Todo ello dificultaba la exclusión del ejército que no estuviera contemplada por la ley (Frieyro de Lara, 2002).

10. Los partes de baja que se encuentran en el Archivo del Círculo Católico de Obreros de Alcoy son una buena muestra de la cantidad de obreros que se quebraban por esa época a consecuencia de los esfuerzos que realizaban.

\section{BIBLIOGRAFÍA}

Alter, George, Neven, Muriel y Oris, Michael (2004), "Stature in Transition. A Micro-Level Study from Nineteenth-Century Belgium", Social Science History, 28 (2), pp. 231-247.

Aracil, Rafael y García-Bonafé, Márius (1974), Industrialització al País Valenciá: el cas d'Alcoy. Valencia, EliseuCliment.

Ayuda, María Isabel y Puche, Javier (2014), “Determinants of heights and biological inequality in Mediterranean Spain, 1859-1967", Economics and Human Biology, 15, pp. 101-119.

Barona, Josep Lluis (2014), La medicalización del hambre. Economía política de la alimentación en Europa, 1918-1960. Barcelona, Icaria Editorial.

Baten, Jorg (2001), "Climate, grain production, and nutritional status in southern Germany during the XVIIIth century", Journal of European Economic History, 30, pp. 9-47.

Baten, Jorg y Blum, Matthias (2014), "Human height since 1820". In: Van Zanden, Jan Luiten et al. (eds.), How Was Life?: Global Well-being since 1820, OECD Publishing. http:// dx.doi.org/10.1787/9789264214262-en

Bell, Frances y Millward, Robert (1998), "Public health expenditures and mortality in England and Wales, 1870-1914", Continuity and Change, 12, pp. 221-249.

Beneito, Ángel (2003), Condicions de vida i salut a Alcoy durant el procésd'industrialització. Valencia, Universitat Politècnica de València.
11. Según el estudio de Beneito (2003, p. 269), durante toda la segunda mitad del siglo XIX destacaron los altos porcentajes de fallecimiento por bronquitis y neumonías respecto al resto de poblaciones rurales de la comarca (un $18 \%$ de muertes frente al $13 \%$ entre 1875 y 1879).

12. En 1884, el precio de algunos productos básicos como el pan, los garbanzos, el aceite, el arroz y la carne de carnero, eran ya más caros en Alcoy que en el resto de poblaciones rurales de la comarca y estaban por encima de la media provincial. Este diferencial, hasta cierto punto, era lógico, ya que los gastos que realizaban los jornaleros y campesinos para adquirir alimentos era inferiores a los que tenían que hacer los trabajadores industriales de Alcoy que no podían autoabastecerse de alimentos básicos como hacían muchas familias campesinas (Beneito, 2003, pp. 63-64).

13. Todavía en 1934, la clínica de la Mutualidad de Levante tramitaría un total de 366 bajas laborales que estaban ocasionadas por heridas contusas (Beneito, 2003, p. 58).

Bernabeu, Josep y Barona, Josep Lluis (2011), Nutrición, salud y sociedad. España y Europa en los siglos XIX y XX. Valencia,Seminari d'Estudis sobre la Ciencia / Universitat de València.

Bourdelais, Patrice (1997), "Histoire de la population, histoire de la médicine et de la santé : cinquante ans d'expérimentations", Dynamis, 17, pp. 17-36.

Calatayud, Salvador (2001), “Economía en transformación. Agricultura e industria en la época contemporánea (18001950)". En: Paul Preston e Ismael Saz (eds.). De la Revolución liberal a la democracia parlamentaria: Valencia (18081975), Valencia, Biblioteca Nueva-Universitat de Valencia, pp. $163-200$

Cage, Robert A. y Foster, John. (2002), "Overcrowding and infant mortality: a tale of two cities", Scottish Journal of Political Economy, 49, pp. 129-149.

Carreras, Albert y Tafunell, Xavier (2010), Historia Económica de la España Contemporánea (1789-2009). Barcelona, Editorial Crítica.

Cinnirella, Francesco (2008), "On the road to Industrialization: nutritional status in Saxony, 1690-1850", Cliometrica, 2 (3), pp. 229-257.

Costa, Dora (2013), "Health and the Economy in the United States, from 1750 to the Present", NBER Working Paper No. 19685 (November). 
Craig, Lee A.; Goodwin, Barry y Grennes, Thomas (2004), "The Effect of Mechanical Refrigeration on Nutrition in the United States", Social Science History, 28 (2), pp. 325-336.

Cutler, David y Miller, Grant (2005), "The Role of Public Health Improvements in Health Advances: The Twentieth-Century United States", Demography, 42, pp. 1-22., [en línea], disponible en: http://muse.jhu.edu/journals/demography/toc/ dem42.1.html, [consultado el 15/10/2014].

Egea, Pedro M. (1984), "La clase obrera en Alcoy a fines del siglo XIX", Anales de Historia Contemporánea, 3, pp. 123-158.

Floud, Roderick; Fogel, Robert W.; Harris, Bernard y Hong, Sok Chul. (2011), The Changing Body: Health, Nutrition, and Human Development in the Western World since 1700. Cambridge, University Press.

Floud, Roderick; Fogel, Robert W.; Harris, Bernard y Hong, Sok Chul. (2014),Health, Mortality and the Standard of Living in Europe and North America since 1700, Volume II, Part I. Cheltenham, UK, Edward Elgar Publishing

Foster, Jim M.; Chinn, Susan y Rona, Robert J. (1983), “The relation of the height of primary school children to population density", International Journal of Epidemiology, 12, pp. 199-203.

Frieyro de Lara, Beatriz (2002), De campesino a soldado. Las quintas en Granada (1868-1898). Granada Universidad de Granada.

García-Gómez, José Joaquín (2013), El nivel de vida de los trabajadores de Alcoy (1836-1936), Tesis doctoral inédita. Alicante, Universidad.

García-Gómez, José Joaquín y Salort, Salvador (2014), "La reforma sanitaria en Alcoi (1836-1914): industrialización, urbanización, fallos de mercado e intervención pública", Historia Social, 80, pp. 95-112.

García-Gómez, José Joaquín (2015), “El nivel de vida de los trabajadores de Alcoy: salarios, nutrición y reforma sanitaria (1836-1913)", Investigaciones de Historia Económica-Economic History Research, 11, pp. 164-173.

Haines, Michael R. (2004), "Growing, Incomes, Shrinking People. Can Economic Development Be Hazardous to Your Health? Historical Evidence for the United States, England, and the Netherlands in the Nineteenth Century", Social Science History, 28 (2), pp. 249-270.

Harris, Bernard (1994): "Health, Height, and History: An Overview of Recent Developments in Anthropometric History", Social History of Medicine, 7 (2), pp. 297-320.

Hatton, Timothy J. (2013), "How have Europeans grown so tall?", Oxford Economic Papers, September, pp. 1-24.

Huck, Paul (1995), "Infant mortality and living standards of English workers during the Industrial Revolution", Journal of Economic History, 55, pp. 531-547.
Humphries, Jane y Leunig, Timothy (2009), "Was Dick Whittington taller than those he left behind? Anthropometric measures, migration and the quality of life in early nineteenth century London?", Explorations in Economic History, 46 (1), pp. 120-131.

Komlos, John (1998), "Shrinking in a Growing Economy? The Mystery of Physical Stature during the Industrial Revolution", Journal of Economic History, 58 (3), pp. 779-802.

Komlos, John y Küchenhoff, Helmut (2012), "The diminution of the physical stature of the English male population in the eighteenth century", Cliometrica, 6, pp. 45-62.

Maluquer, Jordi (2006), "La paradisiacal estabilidad de la anteguerra. Elaboración de un índice de precios de consumo en España, 1830-1936", Revista de Historia Económica, 2, pp. 333-382.

Martínez-Carrión, José Miguel (2012), “La talla de los europeos, 1700-2000: ciclos, crecimiento y desigualdad", Investigaciones de Historia Económica, 8, pp. 176-187.

Martínez-Carrión, José Miguel, y Moreno Lázaro, Javier (2007), "Was there an urban height penalty in Spain, 1840-1913?", Economics and Human Biology, 5, pp. 144-164.

Martínez-Carrión, José Miguel; Puche, Javier y Cañabate, José (2013), "El trabajo infantil y la estatura durante la primera industrialización española, 1840-1930". En: Borderías, Cristina; Borrás Llop, José (eds.), Trabajo infantil y género. BarceIona, Ed. Icaria, pp. 235-272.

Martínez-Carrión, José Miguel; Pérez-Castroviejo, Pedro M; Puche, Javier y Ramon-Muñoz, Josep María (2014), "La brecha rural-urbana de la estatura y el nivel de vida al comienzo de la industrialización española", Historia Social, 80, pp. 35-57.

Martínez Galarraga, Julio (2009), La producción industrial en el País Valenciano (1861-1920). Barcelona, Editorial Milenio.

Mckeown, Thomas (1978), El crecimiento moderno de la población. Barcelona, Antoni Bosch.

Millward, Robert y Baten, Jorg (2010), "Population and living standards 1914-1945". In: Broadberry, Stephen N; O'Rourke, Kevin H. (eds.), The Cambridge Economic History of Modern Europe, vol. 2. Cambridge, University Press, pp. 1914-1945.

Pascual Artiaga, Mercedes y Trescastro, Eva María (2013), El desarrollo del municipio liberal y el reto de la alimentación en el Alicante de la primera mitad del siglo XIX. Alicante, Universidad de Alicante.

Puche, Javier (2009), Evolución de los 'niveles de vida biológi-

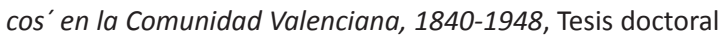
inédita. Sevilla, Universidad Pablo de Olavide.

Puche, Javier (2011), “Evolución de los niveles de vida biológicos en la Comunidad Valenciana, 1840-1948", Investigaciones de Historia Económica, 7 (3), pp. 380-394. 
Ramon-Muñoz, Josep María (2011), “Industrialización, urbanización y bienestar biológico en Cataluña, 1840-1935: una aproximación antropométrica", Revista de Historia Industrial, 46, pp. 41-71.

Reis, Jaime (2009), “'Urban Premium' or 'Urban Penalty'?”, Historia Agraria, 47, pp. 69-94.

Salort, Salvador (2008), Vivir y morir en Alicante. Higienistas e inversiones públicas en salud (1859-1923). Alicante, Universidad.

Silventoinen, Karri (2003), "Determinants of variation in adult body height", Journal of Biosocial Sciences, 35, pp. 263-285.

Steckel, Richard H. (2008), "Biological Measures of the Standard of Living" Journal of Economic Perspectives, 22 (1), pp. 129-152.

Steckel, Richard H. y Floud, Roderick (1997), Health and Welfare during the Industrialization. Chicago and London, The University of Chicago Press.
Szreter, Simon (1988), "The importance of social intervention in Britain's mortality decline, 1850-1914: a reinterpretation of the role of public health, Social History of Medicine, 1, pp. 1-38.

Szreter, Simon (1997), “Economic Growth, Disruption, Deprivation, Disease, and Death: On the Importance of the Politics of Public Health for Development", Population and Development Review, 23 (4), pp. 693-728.

Voth, Hans J. (2004), "Living standards and the urban environment". In: Floud, Roderick; Johnson, Paul (eds.), The Cambridge Economic History of Modern Britain, Volume I: Industrialisation, 1700-1860. Cambridge, University Press, pp. 268-294.

Williams, Naomi y Mooney, Graham (1994), “Infant Mortality in an 'age of Great Cities': London and the English Provincial Cities Compared, c. 1840-1910”, Continuity and Change, 9 (2), pp. 185-212 